\title{
Highly Sensitive Detection of TNT Using a Poly(amidoamine) Dendron-Based SPR Immunosensor
}

\author{
Yutaka Mizuta*, Takeshi Onodera ${ }^{1}$, Praveen Singh ${ }^{2}$, \\ Kiyoshi Matsumoto ${ }^{3}$, Norio Miura ${ }^{4}$ and Kiyoshi Toko ${ }^{1}$ \\ Graduate School of Information Science and Electrical Engineering, Kyushu University, \\ 744 Motooka, Nishi-ku, Fukuoka-shi, Fukuoka 819-0395, Japan \\ 'Faculty of Information Science and Electrical Engineering, Kyushu University, \\ 744 Motooka, Nishi-ku, Fukuoka-shi, Fukuoka 819-0395, Japan \\ ${ }^{2}$ Biophysics and Electron Microscopy Section, Indian Veterinary Research Institute, \\ Izatnagar, Bareilly 243-122, India \\ ${ }^{3}$ Faculty of Agriculture, Kyushu University, \\ 6-10-1 Hakozaki, Higashi-ku, Fukuoka-shi, Fukuoka 812-8581, Japan \\ ${ }^{4}$ Art, Science and Technology Centre for Cooperative Research, \\ Kyushu University, 6-1 Kasuga-kouen, Kasuga-shi, Fukuoka 816-8580, Japan
}

(Received October 5, 2009; accepted April 13, 2010)

Key words: SPR, TNT, PAMAM, dendron, hapten

In this paper, we describe the fabrication of novel poly(amidoamine) (PAMAM) dendron-based Surface Plasmon Resonance (SPR) sensor chip surfaces supported by a self-assembled monolayer (SAM) of alkanethiol and their use in the highly sensitive detection of 2,4,6-trinitrotoluene (TNT), which is one of main components of explosives. The fabricated surfaces showed excellent binding capacity for TNT antibodies; this is probably due to the fact that the highly branched functional groups and three-dimensional structure of dendron molecules offer densely arranged binding sites and improve binding efficiency. A limit of detection (LOD) of ca. $15 \mathrm{ppt}\left(\mathrm{pg} \mathrm{ml}^{-1}\right)$ was achieved by inhibition assay.

\section{Introduction}

There is an increasing concern about terrorism. Portable field deployable sensors capable of highly sensitive, rapid, reliable, and inexpensive detection of explosive substances are strongly demanded for proper on-site inspection, which can contribute to preventing transport of explosives and therefore thwarting terrorist attacks. Surface plasmon resonance (SPR) sensors, which are highly sensitive optical sensors enabling real-time monitoring of refractive index change localized within a few hundred nanometers from thin gold films on their surfaces, ${ }^{(1)}$ can fulfill these requirements; SPR

*Corresponding author: e-mail: mizuta@belab.ed.kyushu-u.ac.jp 
sensors can detect explosive substances not only with high sensitivity but also with high selectivity when used in combination with an antigen-antibody immunoreaction. For the past few years, we developed highly sensitive SPR immunosensors for TNT, TNP, and DNT detection using an inhibition assay.(2-7) In these studies, hapten-protein conjugates were often used as constituent materials of SPR sensor chips ${ }^{(2-4,6)}$ because the threedimensional structure of the surface-bound proteins created an easily accessible binding environment for antibodies, which were flowed over the sensor chip surfaces, and contributed to the enhancement of SPR response.

From the above viewpoint, dendrimers, which are repeatedly branched symmetric compounds with a spherical shape, could be utilized as constituent materials of highly sensitive SPR sensor surfaces because the three-dimensional structure of dendrimers and their densely arranged binding sites can improve the accessibility to the target biological macromolecule. ${ }^{(8,9)}$ Cystamine core poly(amidoamine) (PAMAM) dendrimers are unique among other dendrimers in that they can be divided into two single-site, thiolfunctionalized dendrons by the reduction of the disulfide bonds at their cores. ${ }^{(10)}$ The reactive thiol groups formed at the focal point of the didendron fragments can then be used as single points for reaction with other substances. The application of dendrons for quantitative SPR analysis has yet to be fully investigated. Therefore, we applied dendron-based surfaces for highly sensitive SPR immunoassay of 2,4,6-trinitrotoluene (TNT), one of the main components of explosives.

\section{Experimental Details}

\subsection{Reagents and chemicals}

The following chemicals were used without further purification: mouse anti-TNT monoclonal antibody (TNT antibody) obtained from Strategic Biosolutions, USA; 21.4 $\mu \mathrm{g} \mathrm{ml}^{-1}$ of TNT solution in Milli-Q water purchased from Chugoku Kayaku Co. Ltd., Japan; 2,4-dinitrophenyl acetic acid (DNP-acetic acid) purchased from Tokyo Chemical Industry, Japan; 11-mercaptoundecanoic acid (11-MUA) obtained from Sigma-Aldrich, USA; carboxy-EG6-undecanethiol [20-(11-mercaptoundecanyloxy)-3,6,9,12,15,18hexaoxaeicosanoic acid], and 11-amino-1-undecanethiol, hydrochloride (11-AUT) obtained from Dojindo Laboratory, Japan; 1-ethyl-3-(3'-dimethylaminopropyl)carb odiimide (EDC) and $N$-hydroxysuccinimide (NHS) purchased from GE Healthcare, USA; $N$-[E-maleimidocaproic acid] hydrazide (EMCH), tris(2-carboxyethyl)phosphine, hydrochloride (TCEP), and Ellman's reagent [5,5'-dithio-bis-(2-nitrobenzoic acid)] purchased from Thermo Fisher Scientific, USA; and amine-terminated polyamidoamine (PAMAM) dendrimer, cystamine core generation 3 (G3) with 64 surface primary amino groups $\left(\mathrm{C}_{304} \mathrm{H}_{612} \mathrm{~N}_{122} \mathrm{O}_{60} \mathrm{~S}_{2}\right.$, MW 7,001 in $10 \mathrm{wt} \%$ methanol), and cystamine core generation 6 (G6) with 256 surface primary amino groups $\left(\mathrm{C}_{2544} \mathrm{H}_{5092} \mathrm{~N}_{1018} \mathrm{O}_{508} \mathrm{~S}_{2}\right.$, MW 58,140 in $10 \mathrm{wt} \%$ methanol) purchased from Dendritic Nano Technologies, USA. All other chemicals were purchased from either Tokyo Chemical Industry, Japan or Wako Pure Chemical Industries, Japan. All aqueous solutions were prepared from Milli-Q deionized water obtained from Milli-Q system (Millipore Corporation, USA). 


\subsection{Fabrication of sensor chip surfaces}

SIA Kit Au (GE Healthcare, USA), which contains sensor chips covered with a 50-nm-thick unmodified gold layer, was used for the fabrication of multistep immobilization of various films on surfaces. The sensor chip was cleaned in a mixed solution of Milli-Q water, ammonia solution, and hydrogen peroxide with a 5:1:1 volume ratio at $90^{\circ} \mathrm{C}$ for $20 \mathrm{~min}$. After the cleaning process, the sensor chip was immersed in 1 $\mathrm{mM}$ alkanethiol (in ethanol) for about $24 \mathrm{~h}$ at room temperature to form a self-assembled

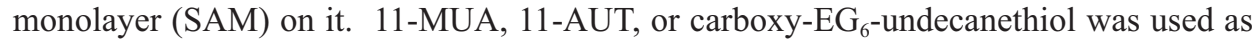
the SAM-forming reagent.

The cystamine-core PAMAM dendrimer was converted into two dendron thiols by reducing the disulfide bond. TCEP, which is one of disulfide-reducing reagents, was used. When using TCEP, there is no need for the desalting (removing excess TCEP after the reaction) step that would be necessary if dithiothreitol (DTT) or another disulfidereducing reagent was used. The G3 or G6 cystamine-core PAMAM dendrimer (in 10 wt $\%$ methanol) was mixed with $50 \mathrm{mM}$ TCEP (in $50 \mathrm{mM}$ Tris $\cdot \mathrm{HCl} \mathrm{pH}$ 7.5, 5mM EDTA) at a 1:4 volume ratio and incubated for about $1 \mathrm{~h}$. The occurrence of thiol reduction was observed using Ellman's reagent on a silica gel TLC plate, as described previously. ${ }^{(10)}$ EDTA plays an important role in maintaining sulfhydryl groups generated by oxidation because sulfhydryl groups are fairly stable in the presence of EDTA.

The chips modified with carboxyl-terminated SAMs (11-MUA or carboxy- $\mathrm{EG}_{6}$ undecanethiol) were immersed in a mixed solution of $0.4 \mathrm{M}$ EDC (in water) and $0.1 \mathrm{M}$ NHS (in water) with a 1:1 volume ratio for $30 \mathrm{~min}$ and the carboxyl terminal groups of the SAMs were converted to NHS esters. Then, the chips were immersed in $10 \mathrm{mM}$ EMCH (in PBST, $\mathrm{pH} 7.2,150 \mathrm{mM} \mathrm{NaCl}$ ) for $1 \mathrm{~h}$; amino terminal groups of EMCH reacted with NHS esters and maleimide groups were introduced into the surface. The obtained chip was immersed in the G3- or G6-PAMAM-dendron-thiol-containing solution for $1 \mathrm{~h}$. Sulfhydryl groups of dendron thiol reacted specifically with maleimide groups of SAM terminals to form irreversible thioether bonds.

For the immobilization of TNT analogues, carboxyl groups of DNP-acetic acid were activated with EDC and NHS solution to form NHS-esterified DNP-acetic acid. Fifty microliters of $10 \mathrm{mM}$ DNP-acetic acid in $N, N$-dimethyl formamide (DMF) was mixed with $50 \mu 1$ of $0.4 \mathrm{M}$ EDC (in water) and $50 \mu \mathrm{l}$ of $0.1 \mathrm{M}$ NHS (in DMF) in a microtube and incubated for about $1 \mathrm{~h}$. The dendron-immobilized sensor chips were immersed in the solution for about $1 \mathrm{~h}$ for the reaction between the amino terminals of the sensor surface and the NHS esters of the DNP-acetic acid. After the reaction, the remaining reactive NHS esters and maleimides were blocked by immersing the chip in $50 \mathrm{mM}$ l-cysteine for $30 \mathrm{~min}$ at room temperature. After rinsing with Milli-Q water, the chips were blown dry under nitrogen and used for SPR experiments.

For the fabrication of a non-dendron-modified chip, an 11-AUT SAM modified chip was immersed in NHS-esterified DNP-acetic acid solution, which was prepared by the same method mentioned above, for about $1 \mathrm{~h}$. After rinsing with Milli-Q water, the chip was blown dry under nitrogen and used for SPR experiments. Figure 1 shows the fabrication procedure of the G6-dendron-based sensor surface. 

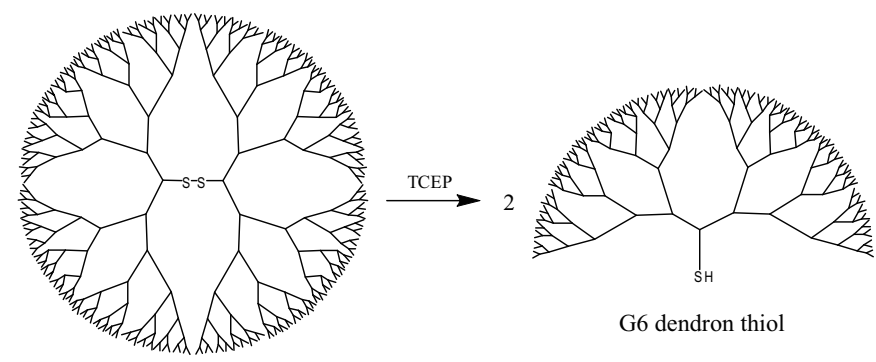

G6 dendron thiol

G6 cystamine core dendrimer

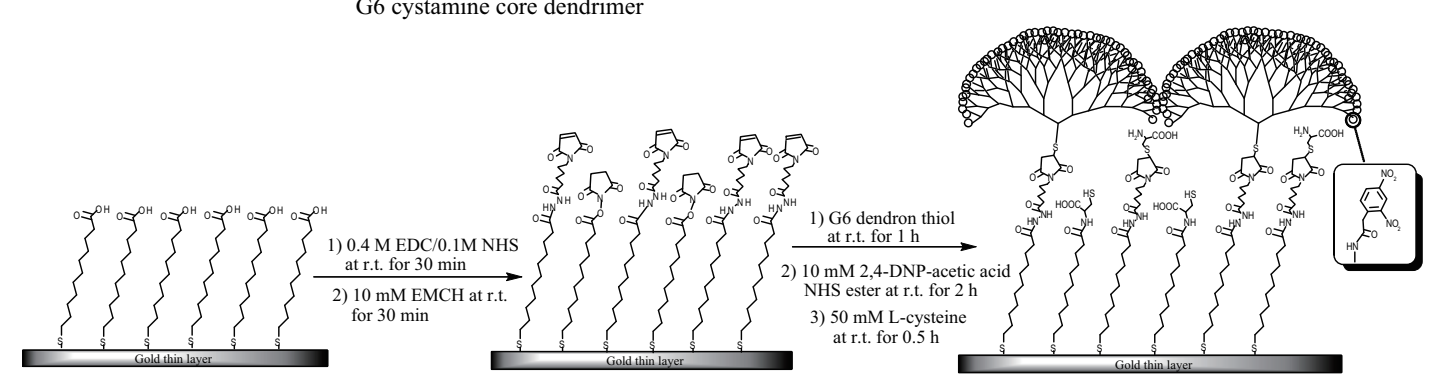

Fig. 1. The fabrication procedures of the G6-dendron-based sensor surface.

\subsection{Interaction analyses using SPR sensor}

\subsubsection{Instrument and conditions}

SPR measurements were carried out using a BIAcore J SPR sensor system. All measurements were conducted at a constant temperature of $25^{\circ} \mathrm{C}$ and a flow rate of $30 \mu \mathrm{l}$ $\min ^{-1}$. An increase in resonance units (RU) corresponds to an increase in the amount of bound antibody on the surface. $1 \mathrm{RU}$ is determined as $0.0001^{\circ}$ of resonance angle shift and is equivalent to a change in mass of $1 \mathrm{pg} \mathrm{mm}^{-2}$ on the surface. ${ }^{(1)} \mathrm{NaOH}$ solution was used for the regeneration of sensor surfaces. In most of the experiments, surface-bound antibodies were completely dissociated when $5 \mathrm{mM} \mathrm{NaOH}$ was flowed over the surfaces. When a surface was not completely regenerated, a higher concentration of $\mathrm{NaOH}$ was used. PBST (100 mM phosphate-buffered saline, $150 \mathrm{mM} \mathrm{NaCl}, 0.05 \%$ (v/v) Tween 20, $\mathrm{pH}$ 7.2) was used as a running buffer.

\subsubsection{SPR detection of TNT}

TNT detection was carried out by inhibition assay.(1) A fixed concentration of 80 $\mathrm{ng} \mathrm{ml}^{-1}$ (ppb) TNT antibody solution was mixed with various concentrations of TNT solution (2 $\mathrm{pg} \mathrm{ml}^{-1}$ (ppt), $20 \mathrm{ppt}, 200 \mathrm{ppt}, 2 \mathrm{ng} \mathrm{ml}^{-1}$ (ppb), and $20 \mathrm{ppb}$ ) in 1:1 volume ratio and incubated at room temperature for about $15 \mathrm{~min}$ before injection. Thus, the final concentration of TNT antibody in each sample solution was $40 \mathrm{ppb}$, and those of TNT were $1,10,100,1$, and $10 \mathrm{ppb} .210 \mu \mathrm{l}$ of each sample solution ( 7 min injection) was injected more than 3 times and the median of the data was used for calibration. Each sample solution was prepared with PBST. 


\section{Results and Discussion}

\subsection{Binding capacity of dendron-immobilized surfaces}

To examine the binding capacity of the dendron-immobilized surface and compare it with the non-dendron-modified surface or the higher (lower)-generation dendronimmobilized surface, three types of sensor surfaces ((a) G6-dendron-based surface, (b) G3-dendron-based surface, and (c) non-dendron-modified surface) were fabricated using the procedure shown in $\S 2.2$. The surface architectures of the chips are as follows: (a) DNP-acetic acid + G6 dendron + 11-MUA; (b) DNP-acetic acid + G3 dendron + 11-MUA; and (c) DNP-acetic acid + 11-AUT. Sixty microliters each of TNT antibody solutions of $0.2,0.4,1,2,4,10,20$, and $40 \mu \mathrm{g} \mathrm{ml}^{-1}$ (ppm) was flowed over surfaces (a), (b), and (c) (2 min each). The experimental result is shown in Fig. 2. The G6-dendron-based surface (a) shows a 2.4-fold higher binding response than the non-dendron-modified surface (c) in all measurements. The G3-dendron-based surface (b) shows a 1.8-fold higher binding response than the non-dendron-modified surface (c) in all measurements. Figure 2 also shows that a higher sensor response is observed on the G6-dendron-based surface (a) than on the G3-dendron-based surface (b). This suggests that the amount of sensor response depends strongly on the density of surface-bound haptens. It is also explained by the three-dimensional structure of dendron molecules.

\subsection{Highly sensitive detection of TNT by inhibition assay}

Small molecules like TNT (MW < 1,000) cause very small signal changes upon binding to an SPR sensor surface. Hence, an inhibition assay is often used to achieve their highly sensitive detection. In an inhibition assay, ${ }^{(1)}$ small molecular antigens (or their analogues) are immobilized on a sensor surface and antibodies are initially

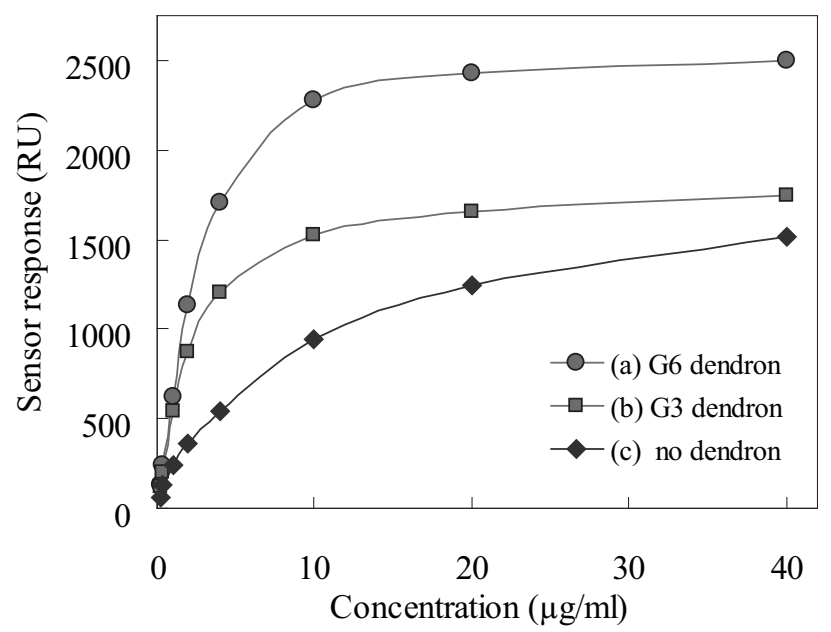

Fig. 2. Binding response of SPR signal on three types of surfaces: (a) G6-dendron-based surface; (b) G3-dendron-based surface; and (c) non-dendron-modified surface. 
mixed with their antigens at a certain concentration. When the mixture is flowed over the surface, only free antibodies can bind to the sensor surface. The reduction in the intensity of the signal decreases linearly with an increase in the amount of antigen in a sample solution. Thus, the amount of antigen can be determined indirectly. In the inhibition assay, the limit of detection (LOD) decreases as antibody concentration decreases; ${ }^{(3,5)}$ thus, it is necessary to use as low a concentration of antibody as possible for highly sensitive detection. According to the result in the previous section, the G6dendron-based surface is the most suitable for TNT detection because it minimizes the analyte concentration required to produce a detectable signal.

The TNT antibody used in this work was raised in mice against the TNP-glycineKLH conjugate as the immunogen. It is one of the most sensitive commercially available monoclonal TNT antibodies: the affinity constant of the antibody is $(1.3 \pm 0.1) \times 10^{9} \mathrm{~L} \mathrm{~mol}^{-1}{ }^{(12)}$ Among other TNT analogues, DNP-acetic acid was chosen as the immobilized hapten in accordance with our previous study. ${ }^{(5)}$

One way to obtain further increase in sensitivity would be to reduce experimental error. Thus, we chose to use carboxy-EG6-undecanethiol, which is an oligo(ethylene glycol) (OEG)-containing reagent, as the SAM-forming reagent instead of 11-MUA because OEG-containing molecules are known to resist protein adsorption owing to their flexibility in the aqueous phase and hydrophilic properties, ${ }^{(13)}$ which enabled reproducible measurements to be made.

Overlayed SPR sensorgrams shown in Fig. 3 show experimental data of the inhibition assay. Each sample solution contains only $40 \mathrm{ppb}$ TNT antibody. However, sufficient sensor responses for quantitative inhibition assay were observed without any

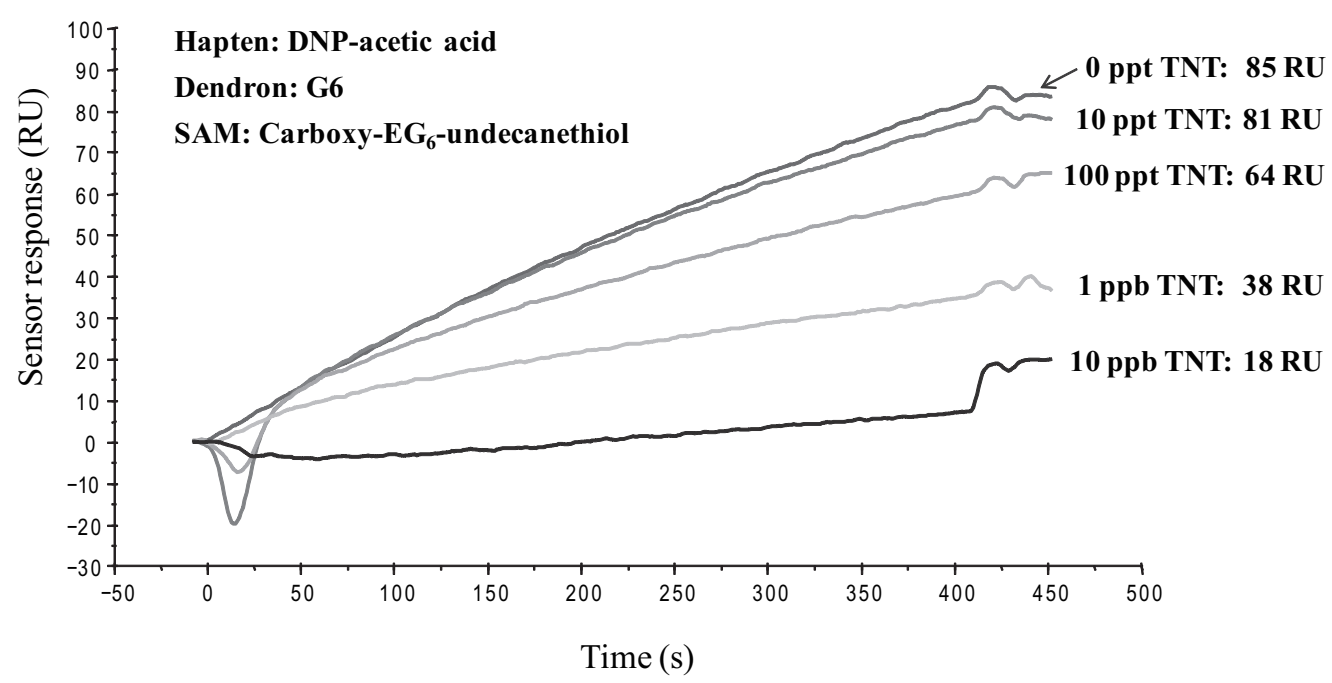

Fig. 3. SPR sensorgrams of inhibition assay using $40 \mathrm{ng} \mathrm{ml^{-1 }} \mathrm{TNT}$ antibody for detection of various concentrations of TNT on DNP-acetic-acid-modified G6-dendron-immobilized surface. Carboxy-EG $\mathrm{E}_{6}$-undecanethiol was used as the SAM-forming reagent. 
signal amplification like those of secondary antibodies. ${ }^{(5,14)}$ Lower sensor responses are obtained at higher TNT concentrations. This shows that the inhibition of TNT antibody binding to immobilized haptens on the surface occurs more frequently at a high concentration of TNT.

Figure 4 shows a calibration curve obtained by four-parameter logistic fit ${ }^{(15)}$ using BIAevaluation software (ver. 3.2, BIAcore). The vertical axis shows bound percentage, which denotes the relative percentage of sensor response of the TNT-containing antibody solution to the antibody solution in the absence of TNT $\left(\Delta \theta / \Delta \theta_{0} \times 100 ; \Delta \theta_{0}\right.$ is the sensor response (RU) for the $40 \mathrm{ppb}$ antibody solution without TNT, which serves as the reference, $\Delta \theta$ is the sensor response (RU) for a mixed solution of $40 \mathrm{ppb}$ antibody and a certain concentration of TNT). The reference solution was measured three times in a cycle of measurements. Bound percentage was calculated using the median of the three measurements. The error bar shows the standard deviation (SD) of the data. The $\mathrm{SD}$ of the reference sample solution was about $2.5 \%$. The limit of detection (LOD) was determined to be about $15 \mathrm{ppt}$ as the TNT concentration yielding $92.5 \%$ bound percentage by considering three SDs of the reference solution.

Thus far, we have confirmed that the G6-dendron-based sensor surface demonstrates excellent performance for the sensitive detection of TNT. The merits of the G6-dendronbased surface are that a small amount of antibody could be used without the need for any signal amplification process and as a result short time detection is possible. Therefore, it could be utilized for the development of portable explosive sensors with high sensitivity and fast detection capability.

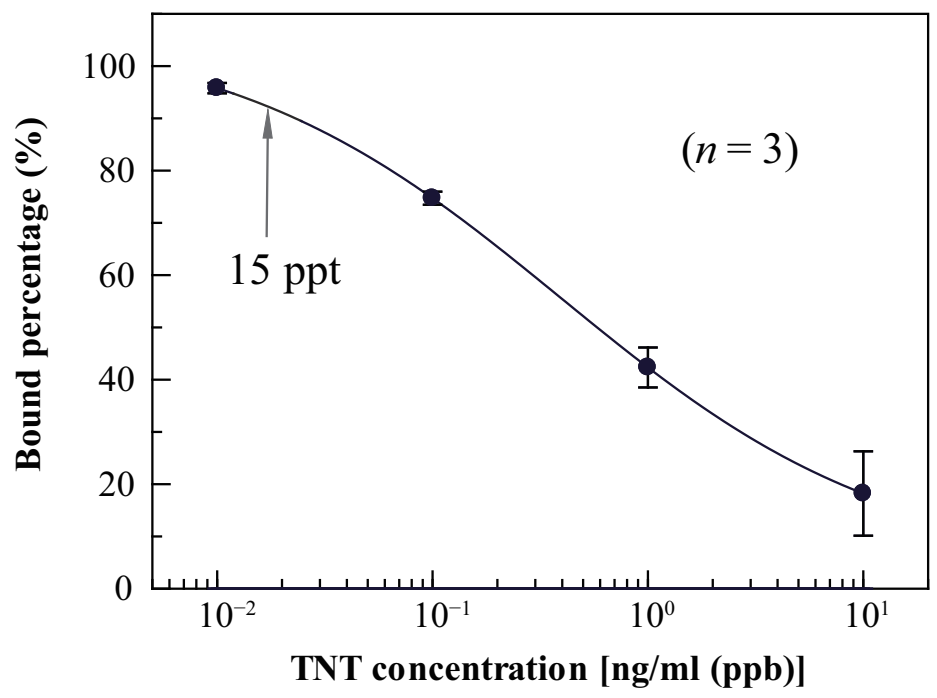

Fig. 4. Calibration curves obtained by inhibition assay using $40 \mathrm{ng} \mathrm{ml}^{-1} \mathrm{TNT}$ antibody. The error bar shows the SD of the data. 


\section{Conclusions}

In this work, we fabricated novel PAMAM dendron-based SPR sensor surfaces supported by a SAM of alkanethiol for the highly sensitive detection of TNT, which is one of the main components of explosives. The fabricated surfaces showed excellent binding capacity for the TNT antibody; the G6-dendron-based surface shows a 2.4-fold higher binding response than the non-dendron-modified surface throughout all measurements. This is probably due to the fact that highly branched functional groups and the three-dimensional structure of dendron molecules offer densely arranged binding sites and improve binding efficiency. Therefore, it can minimize the analyte concentration required to produce a detectable signal. An inhibition assay for TNT detection was conducted on the fabricated G6-dendron-based surface using $40 \mathrm{ppb}$ antibody. An LOD of ca. 15 ppt was achieved without further signal amplification.

\section{Acknowledgements}

This work was partially supported by Japan Science and Technology through Core Research for Evolutional Science and Technology (CREST).

\section{References}

1 J. Homola: Anal. Bioanal. Chem. 377 (2003) 528.

2 N. Miura, D. R. Shankaran, T. Kawaguchi, K. Matsumoto and K. Toko: Electrochemistry 75 (2007) 13.

3 K. Matsumoto, A. Torimaru, S. Ishitobi, T. Sakai, H. Ishikawa, K. Toko, N. Miura and T. Imato: Talanta 68 (2005) 305.

4 P. Singh, T. Onodera, Y. Mizuta, K. Matsumoto, N. Miura and K. Toko: Sensors and Materials 19 (2007) 261.

5 Y. Mizuta, T. Onodera, P. Singh, K. Matsumoto, N. Miura and K. Toko: Biosens. Bioelectron. 24 (2008) 191.

6 D. R. Shankaran, K. V. Gobi, K. Matsumoto, T. Imato, K. Toko and N. Miura: Sens. Actuators, B 100 (2004) 450.

7 K. Nagatomo, T. Kawaguchi, N. Miura, K. Toko and K. Matsumoto: Talanta 79 (2009) 1142.

8 S. S. Mark, N. Sandhyarani, C. Zhu, C. Campagnolo and C. A. Batt: Langmuir 20 (2004) 6808.

9 M.-Y. Hong, D. Lee and H.-S. Kim: Anal. Chem. 77 (2005) 7326.

10 D. A. Tomalia, B. Huang, D. R. Swanson, H. M. Brothers, II and J. W. Klimash: Tetrahedron 59 (2003) 3799.

11 E. Stenberg, B. Persson, H. Roos and C. Urbaniczky: J. Colloid Interface Sci. 143 (1991) 513.

12 A. Zeck, M. G. Weller and R. Niessner: Fresenius J. Anal. Chem. 364 (1999) 113.

13 R. R. Siegel, P. Harder, R. Dahint, M. Grunze, F. Josse, M. Mrksich and G. M. Whiteside: Anal. Chem. 69 (1997) 3321.

14 J. S. Mitchell, Y. Wu, C. J. Cook and L. Main: Anal. Biochem. 343 (2005) 125.

15 J. W. A. Findlay, W. C. Smith, J. W. Lee, G. D. Nordblom, I. Das, B. S. DeSilva, M. N. Khan and R. R. Bowsher: J. Pharm. Biomed. Anal. 21 (2000) 1249. 